\title{
iPSC-derived endothelial cell response to hypoxia via SDF1a/CXCR4 axis facilitates incorporation to revascularize ischemic retina
}

\author{
Hongkwan Cho, ${ }^{1}$ Bria L. Macklin, ${ }^{2}$ Ying-Yu Lin, ${ }^{2}$ Lingli Zhou, ${ }^{1}$ Michael J. Lai, ${ }^{1}$ Grace Lee, ${ }^{1}$ \\ Sharon Gerecht, ${ }^{2}$ and Elia J. Duh' \\ 1Department of Ophthalmology, School of Medicine, Johns Hopkins University, Baltimore, Maryland, USA \\ ${ }^{2}$ Department of Chemical and Biomolecular Engineering, Institute for NanoBioTechnology, Johns Hopkins University \\ Baltimore, Maryland, USA.
}

\begin{abstract}
Ischemic retinopathies are major causes of blindness worldwide. Local hypoxia created by loss of vascular supply leads to tissue injury and aberrant neovascularization in the retina. There is a great need for therapies that enhance revascularization of hypoxic neuroretinal tissue. To test the therapeutic feasibility of human-induced pluripotent stem cell-derived endothelial cells (hiPSC-ECs) for the treatment of ischemic retinopathies, we compared the angiogenic potential of hiPSC-ECs with mature human retinal endothelial cells (HRECs) in response to hypoxia. hiPSC-ECs formed more robust and complex vascular networks in collagen gels, whereas HRECs displayed minimal sprouting. The cells were further tested in the mouse oxygen-induced retinopathy (OIR) model. Retinas with hiPSC-EC injection showed colocalization with host vessels, whereas HRECs lacked such responses. hiPSC-ECs markedly reduced vaso-obliteration and pathological neovascularization. This beneficial effect of hiPSC-ECs was explained by the stromal cell-derived factor-1a (SDF1a)/CXCR4 axis; hiPSC-ECs exhibited much higher cell-surface expression of CXCR4 than HRECs and greater chemotaxis toward SDF1a-embedded 3D collagen hydrogel. Furthermore, treatment with neutralizing antibody to CXCR4 abolished recruitment of hiPSCs in the OIR model. These findings suggest superior angiogenic potential of hiPSC-ECs under hypoxia and underscore the importance of SDF1a/CXCR4 in the reparative function of hiPSC-ECs in ischemic diseases.
\end{abstract}

Authorship note: $\mathrm{HC}$ and BLM are c0first authors and contributed equally to this work.

Conflict of interest: The authors have declared that no conflict of interest exists.

Copyright: () 2020, American Society for Clinical Investigation.

Submitted: July 19, 2019

Accepted: February 20, 2020

Published: March 26, 2020.

Reference information: /CI Insight. 2020;5(6):e131828.

https://doi.org/10.1172/jici.

insight.131828.

\section{Introduction}

Hypoxic microenvironments $\left(<5 \% \mathrm{O}_{2}\right)$ develop when vasculature is regressed after injury or disease progression, leading to ischemia of the tissue. In these ischemic conditions, HIFs, transcription factors, are stabilized and regulate the expression of numerous proangiogenic factors, including VEGF and basic FGF (bFGF), proliferation, and the production of ECM degrading MMPs $(1,2)$.

A critical aspect of ischemic diseases is the need to promote restoration of the vascular supply. This challenge is pivotal for tissue health in a wide array of diseases, including stroke and ischemic retinopathies, disease conditions in which revascularization of ischemic neural tissue is often inadequate (3). Therapeutic strategies enabling more rapid revascularization would greatly reduce ischemia-induced tissue damage.

Endothelial cells play a crucial role in maintaining the integrity of blood vessels and are critical components in vascular regeneration therapy. However, acquiring a sufficient amount of autologous ECs for use therapeutically is difficult (4). Unfortunately, in uncontrolled diabetic patients, chronic hyperglycemia can cause dysfunction and abnormalities in endothelial progenitor cells (EPCs), likely aggravating retinopathy $(5,6)$. Thus, although collecting EPCs from patients is an attractive approach, because it circumvents the need for immunosuppression, it is not a feasible option with which to treat diabetic patients (5). The use of hiPSCs offers the potential to bypass these issues. Recent advances in induced pluripotent stem cell (iPSC) technology has allowed for robust procurement of vascular derivatives from stem cells sources. These iPSC-ECs offer a unique opportunity as an inexhaustible, constant source from which to generate cells for vascular therapies (7). 
The mouse model of OIR is a well-established model of retinal ischemia and pathologic neovascularization (NV) and is the most widely used animal model for ischemic retinopathies, including retinopathy of prematurity (ROP), proliferative diabetic retinopathy, and retinal vein occlusions, which are major causes of blindness globally (8). This model replicates two essential features of ischemic retinopathies: loss of vascular supply and consequent pathologic preretinal NV. In the mouse OIR model, neonatal pups are exposed to a high concentration of oxygen (75\%) for 5 days from P7 to P12, after which time they are brought back to room air $\left(21 \% \mathrm{O}_{2}\right)(8)$. The initial hyperoxic stimulus leads to retinal vaso-obliteration (VO), so that the subsequent lower concentration of oxygen in room air is perceived as a relatively hypoxic environment by the ischemic retina, leading to abnormal pathologic preretinal NV (neovascular tufts) anterior to the retina. This model has emerged as a highly suitable model for investigations of revascularization of ischemic neural and retinal tissue, including therapeutic strategies. In this study, the mouse model of OIR was used as an in vivo model for phenotypic analysis of cellular responses to hypoxia in hiPSC-derived ECs (hiPSC-ECs) as well as a means for accomplishing revascularization through homing and integration in the setting of ischemia.

The use of iPSC-derived cells in ischemic retinal conditions has received very little attention and mechanisms of action of iPSC-EC are lacking. In this context, the suitability of iPSC-derived ECs is a promising and important avenue of investigation, including the behavior of these cells in a hypoxic tissue environment compared with other endothelial cell populations.

Here, we present the first report to our knowledge demonstrating the advances of using hiPSC-ECs over mature human retina EC (HREC) alternatives for therapeutic use in hypoxic settings, both in vitro and in the OIR mouse model. Additionally, we propose an important mechanism for the superiority of hiPSC-EC via SDF1a/CXCR4 axis. We show that hiPSC-ECs, compared with mature HRECs, have improved angiogenic responses, characterized by increased proliferation, network formation, and chemotaxis toward SDF1a under hypoxic conditions in vitro. In addition, intravitreal injection of hiPSC-ECs shows better integration into host tissue, enabling vascular recovery in the mouse model of OIR. Importantly, when the hiPSC-ECs were treated with CXCR4 blocking antibody, their presence within the retina and integration was significantly impaired, highlighting the role of SDF1a/CXCR4 axis during this process.

\section{Results}

hiPSC-EC and HREC response to hypoxia in vitro. Toward determining the opportunities to use hiPSC-ECs for retinal revascularization applications, we first analyzed their phenotypic responses to hypoxia in vitro. To assess the differences in qualitative functionalities of hiPSC-ECs with primary endothelial cells, we used primary HRECs as a mature cell comparison group and exposed both cell types to $1 \%$ oxygen over a 24-hour period. Qualitatively, hiPSC-ECs and HRECs displayed no difference in the expression of the platelet EC adhesion molecule, CD31, or vascular endothelial cadherin (VE-Cad) between atmospheric and hypoxic culture conditions (Figure 1A). Stains and quantification of the proliferation marker Ki67 revealed a significant decrease in HREC proliferation under hypoxia, whereas hypoxia had no statistically significant effect on the proliferation of hiPSC-ECs (Figure 1, B and C).

We next examined HIF accumulation in hiPSC-ECs and HRECs. We found no differences in HIF1A and HIF2A protein accumulation under hypoxic conditions (Supplemental Figure 1; supplemental material available online with this article; https://doi.org/10.1172/jci.insight.131828DS1), suggesting that downstream angiogenic pathways are activated in both cell types. Indeed, when we measured the mRNA regulation of key angiogenesis growth factors $V E G F$ and $b F G F$, we found that, in both cell types, hypoxia resulted in a downregulation of $b F G F$ and an upregulation in $V E G F$ mRNA at similar levels (Figure 1D).

Hypoxic network formation on hiPSC-ECS and HRECS in vitro. To determine the effect of hypoxia on network formation, we next analyzed the vasculogenic potential of hiPSC-ECs and HRECs by using an in vitro 3D collagen hydrogel assay. hiPSC-ECs and HRECs were encapsulated in collagen hydrogel and exposed to hypoxia for 48 hours. In atmospheric conditions, hiPSC-ECs formed robust and complex vascular networks, whereas HRECs displayed some sprouting, with many cells remaining globular after 48 hours (Figure 2A). In hypoxic conditions, hiPSC-EC networks were elongated, with a higher number of vascular tubes quantified (Figure 2B), whereas HRECs formed shorter, less thick tubes (though not statistically significant). In addition, there was a statistically significant reduction in total tube volume of HRECs under hypoxia, highlighting the contrast in hypoxic network formation between hiPSC-ECs and HRECs. 
$\mathbf{A}_{\text {hiPSC-ECs }}$
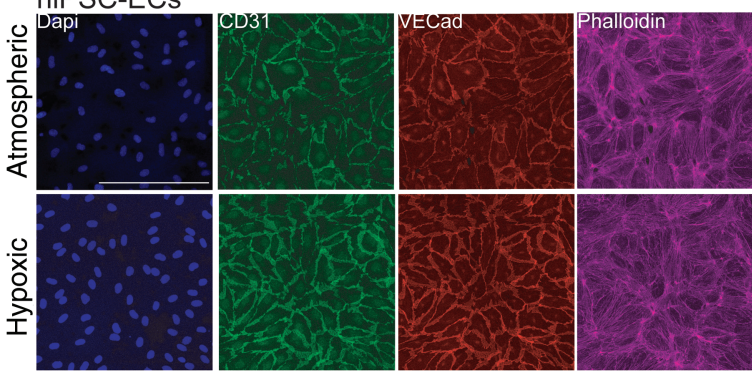

HRECS
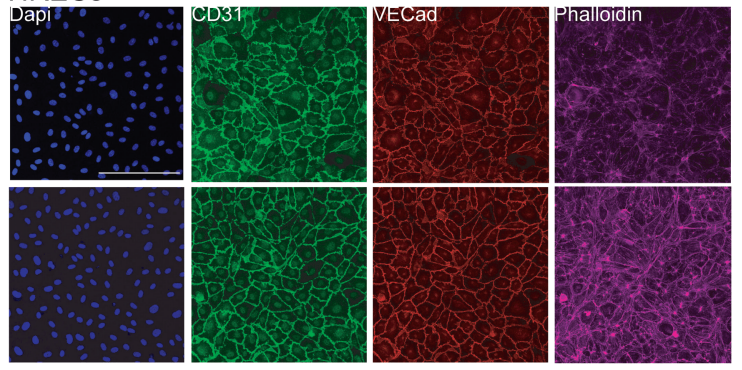

B

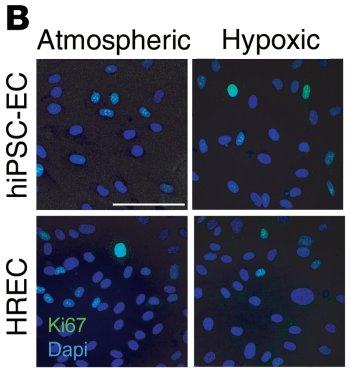

C

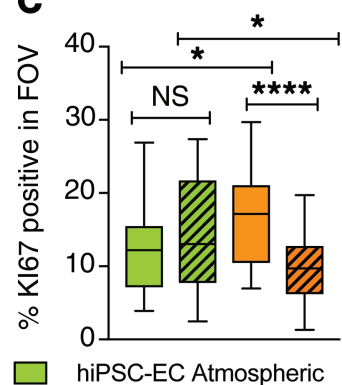

\section{D}
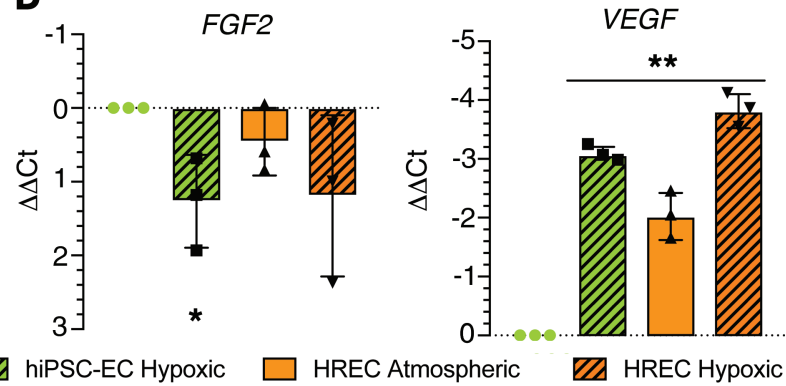

Figure 1. In hypoxia, HRECs show a significant decrease in proliferation compared with hiPSC-ECs. (A) Representative immunofluorescence images of CD31 (green), vascular endothelial cadherin (red), and phalloidin (magenta) are shown. (B) Representative immunofluorescence images of Ki67 staining and (C) quantification. Tukey's multiple comparisons test was conducted to determine statistical significance $(n=30, n=3)$. Box extends from the 25 th to 75 th percentile with median line in the middle; whiskers represent min to max range. (D) qRT-PCR for VEGF and FGF2 normalized to hiPSC-EC atmospheric condition $(n=3)$. Data are presented as mean \pm SD. $t$ test was used to determine statistical significance. Significance levels are set at ${ }^{*} P \leq 0.05,{ }^{*} P \leq 0.01$, and ${ }^{* * * *} P \leq 0.0001$. Scale bars: $200 \mu \mathrm{m}$. HREC, human retinal endothelial cell, hiPSC-EC, human-induced pluripotent stem cell-derived endothelial cell.

hiPSC-ECs integrate into mouse host vasculature in the OIR model. To determine if hiPSC-ECs can successfully integrate into host vasculature in an ischemic setting, we used the OIR model in NOD/SCID mice. We reasoned that the mouse OIR model is an excellent model for studying homing and incorporation of hiPSC-ECs in ischemic/hypoxic retina. Given the translational implications, we used the intravitreal injection approach because intravitreal injections are now the most commonly performed ophthalmic procedure (9). The hiPSC-ECs were injected via the intravitreal route on P12, immediately after removal of pups from $75 \% \mathrm{O}_{2}$, and retinas were collected on P17 (Figure 3A). We found that hiPSC-ECs showed successful colocalization with host vasculature as early as P14 (Supplemental Figure 2), with continued colocalization on P17. In contrast, eyes injected with HRECs resulted in the deposition of cells anterior to the retinal vasculature (Figure 3, B and C). High-magnification images further demonstrated that hiPSC-ECs showed incorporation into regenerating retinal vessels, whereas HRECs showed limited integration at the same time point (Figure 3C). With the results demonstrating incorporation of hiPSC-ECs, but not HRECs, into retinal vasculature, vascular rescue effects of the hiPSC-ECs were evaluated. For this, VO (avascular retinal area) and pathological retinal NV were measured on P17. Intravitreal injection of hiPSC-ECs significantly reduced VO and NV compared with the contralateral eyes injected with PBS (Figure 3D), indicating a marked degree of reparative angiogenesis. In association with this vascular recovery and reduction in ischemic retinal area, treatment with hiPSC-EC resulted in a marked reduction in the pathologic retinal neovascular tufts (Figure 3D). In addition to the immunodeficient NOD/SCID mice, additional studies were performed in the immunocompetent $\mathrm{C} 57 \mathrm{BL} / 6 \mathrm{~J}$ mice and yielded similar results. Intravitreal injection of hiPSC-EC resulted in substantial colocalization with the host retinal vasculature compared with HREC (Supplemental Figure 3A). As in NOD/SCID, intravitreal injection of hiPSC-EC significantly rescued VO and suppressed pathologic retinal NV in C57BL/6J (Supplemental Figure 3B).

Differential response to SDF1a-mediated angiogenesis. To determine the underlying mechanism by which hiPSC-ECs better integrate with the host vasculature in OIR, we examined the SDF1a/CXCR4 pathway. SDF1a is a chemokine that plays a major role in EC recruitment to the secretion site, by binding to the cell membrane bound receptor CXCR4 (10). We began by testing the 3D functional response to SDF1a in both cell types in atmospheric and hypoxic conditions. Toward this, SDF1a was embedded within 
A
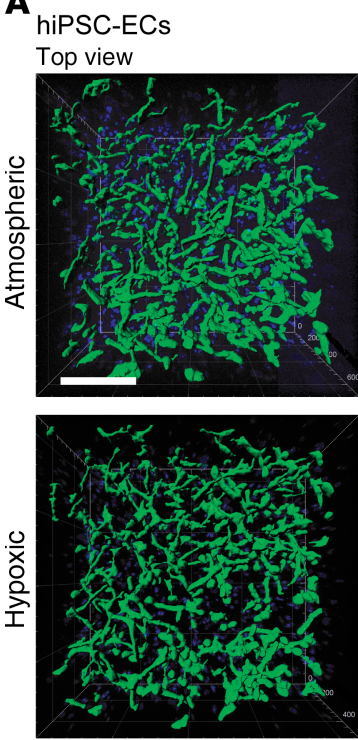

Side view
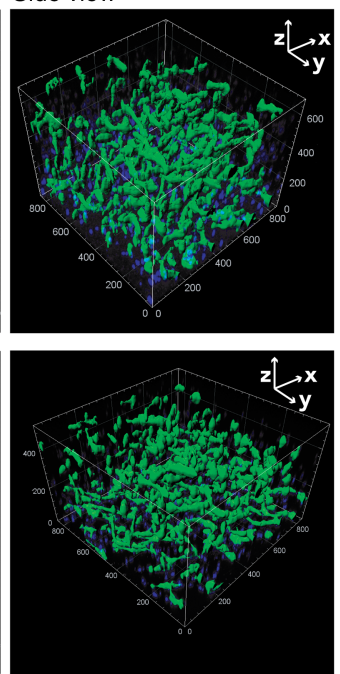

HRECS

Top view
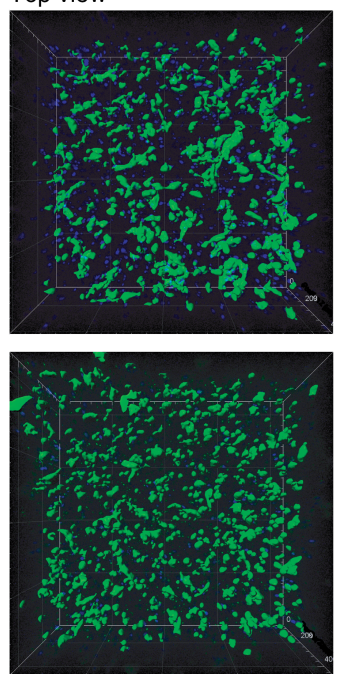

Side view
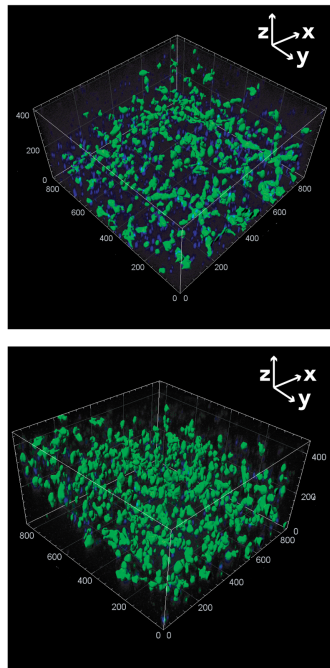

B

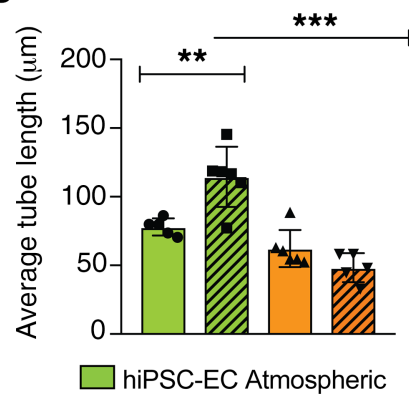

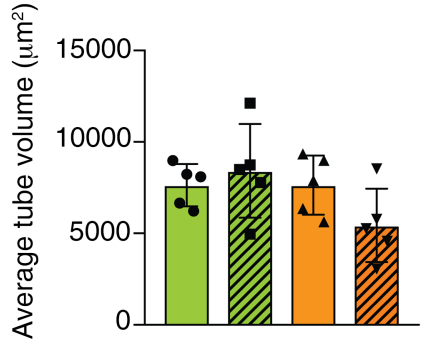

VIA hiPSC-EC Hypoxic

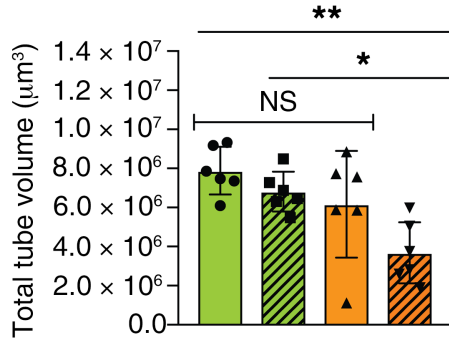

VIA HREC Hypoxic

Figure 2. In hypoxia, hiPSC-ECs form robust vascular networks compared with HRECs. (A) Representative confocal images of human-induced pluripotent stem cell-derived endothelial cells (hiPSC-ECs) or human retinal endothelial cells (HRECs) in collagen type I hydrogels after 48 hours of culture in atmospheric or hypoxic conditions; left panel is a top view and right panel is a side view (phalloidin in green, nuclei in blue, scale bar represents $100 \mu \mathrm{m}$ ). (B) Quantification of average tube length, and tube volume $(n=3)$. Tukey's multiple comparisons test was conducted to determine statistical significance. Significance levels are set at ${ }^{*} P \leq 0.05$, ${ }^{* *} P \leq 0.01$, and ${ }^{* * *} P \leq 0.001$. Data are presented as mean \pm SD. Scale bar: $200 \mu \mathrm{m}$.

collagen type I hydrogels at a concentration of $100 \mathrm{ng} / \mathrm{mL}$, and either hiPSC-ECs or HRECs were seeded on top of the polymerized hydrogels. Invasion into the hydrogel was analyzed after 48 hours. hiPSC-ECs were able to invade the hydrogel similarly in atmospheric and hypoxic conditions. HRECs did not invade the hydrogels in atmospheric conditions but some invasion was observed in hypoxic conditions (Figure 4, A and B). We next used flow cytometry to examine the expression of CXCR4 on the surface of the cells and found high expression levels of the receptor on the hiPSC-ECs in both atmospheric and hypoxic conditions. HRECs displayed a range of CXCR4 expression in atmospheric conditions; however, under hypoxia this shifted and many of the cells lacked expression of CXCR4. (Figure 4C). To confirm the importance of this pathway in vivo, we examined whether the inhibition of CXCR4 modulated hiPSC-EC recruitment in OIR. Intravitreal injection was performed with hiPSC-ECs treated with CXCR4 blocking antibody or vehicle (control). Although there was robust colocalization of control hiPSC-ECs with host vasculature in OIR ( $n=4$ mice), we found no colocalization of hiPSC-ECs treated with CXCR4 blocking antibody ( $n=7$ mice; Figure $4 \mathrm{D})$.

\section{Discussion}

Approximately $10 \%$ of the US population is currently living with diabetes mellitus (11). Hallmarks of the disease, including hyperglycemia, increased fatty acids, and oxidative stress, individually and collectively lead to vascular damage (12). Hypoxia, caused by lack of adequate $\mathrm{O}_{2}$ supply to retinal vessels, 
A

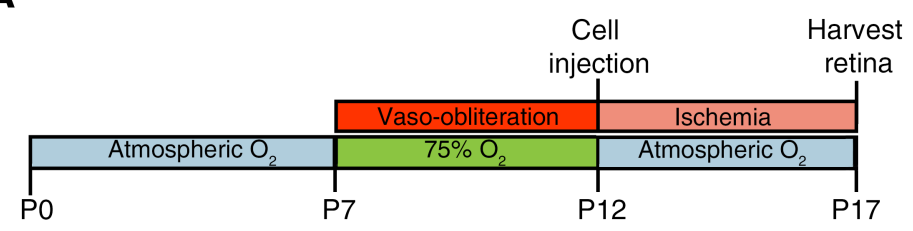

B
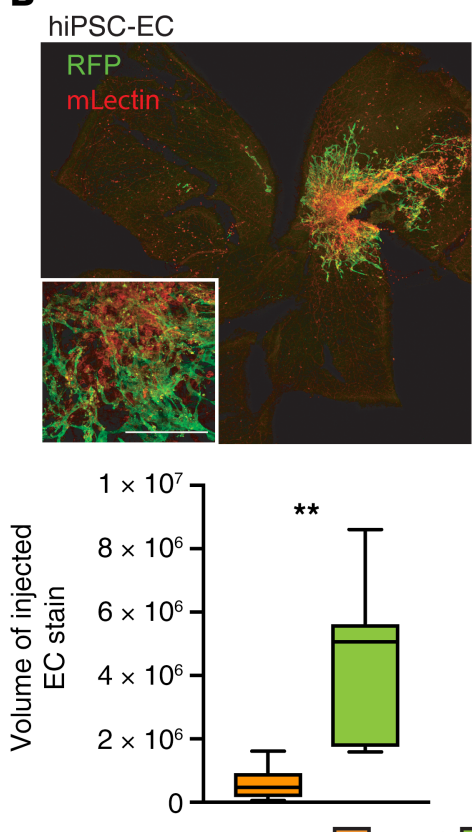

HREC
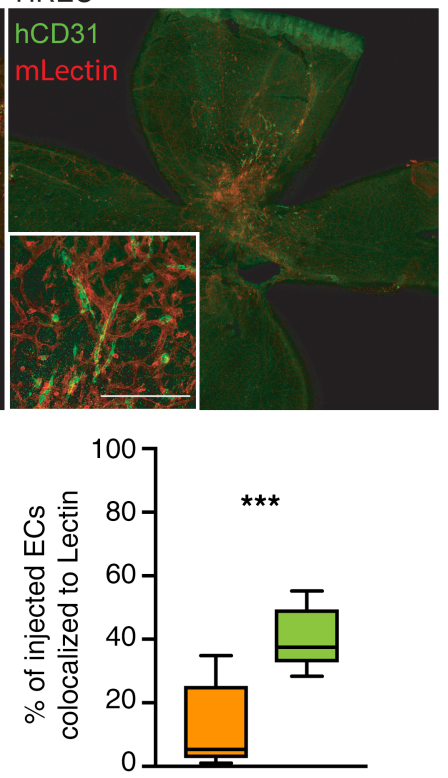

$\square$ HREC $\square$ hiPSC-EC

C

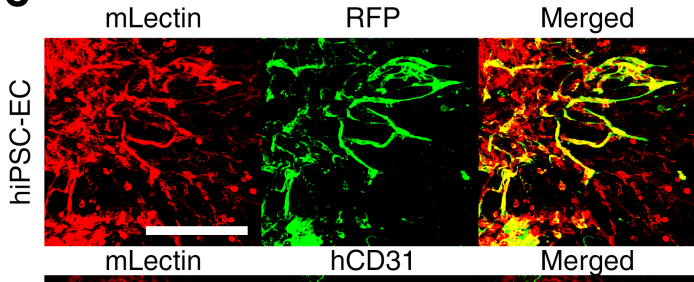

mLectin

RFP Merged

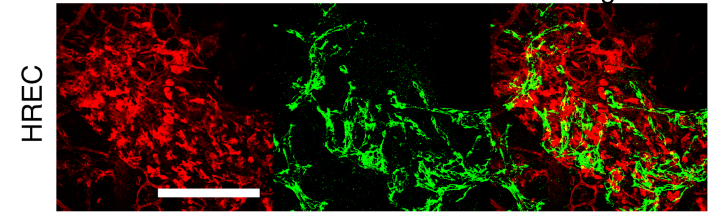

D
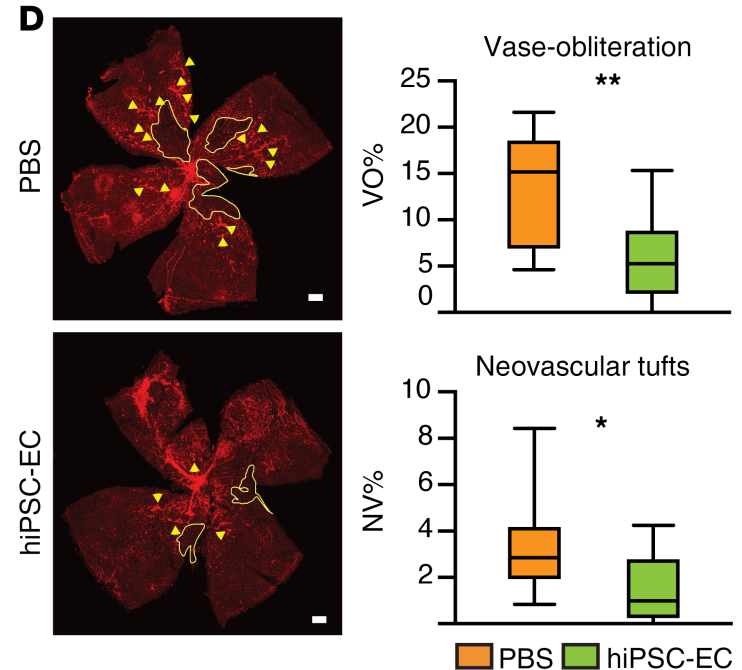

Figure 3. hiPSC-ECs exhibit colocalization with host vasculature and accelerate vascular recovery in a mouse model of oxygen-induced retinopathy. (A) Schematic diagram of experimental protocol of oxygen-induced retinopathy (OIR) in mouse. (B) Representative immunofluorescence images of the mouse retinal tissue at OIR P17, 5 days after intravitreal injection of human-induced pluripotent stem cell-derived endothelial cells (hiPSC-ECs) or human retinal endothelial cells (HRECs) with quantifications of cell volume and \% colocalization to host lectin staining $(n=7)$. (C) Representative images showing colocalization of hiPSC-ECs (top) and HRECs (bottom) with host vasculature (lectin, red). (D) Vascular rescue by hiPSC-ECs in OIR. Both VO area (outlined in yellow) and pathological neovascularization (yellow arrows) were significantly decreased at P17 $(n=12)$. Box extends from the 25th to 75 th percentile with median line in the middle; whiskers represent minimum to maximum range. Significance levels are set at ${ }^{*} P \leq 0.05,{ }^{*} P \leq 0.01$, and ${ }^{* * *} P \leq 0.001$ by 2-tailed Student's $t$ test. Scale bars: $200 \mu \mathrm{m}$. RFP, red fluorescent protein.

induces a cascade of signaling events, exacerbating the disease from the earlier nonproliferative stage to proliferative diabetic retinopathy (DR), which is a major cause of blindness (13). Human PSC derivatives offer a goldmine of therapeutic prospect for regenerative medicine. hiPSC-ECs have been shown to restore perfusion in hind limb ischemia models $(14,15)$ and accelerate wound healing $(16-18)$, making them an ideal source for the treatment of vascular diseases, including diabetic retinopathy. hiPSC-ECs tout similar benefits and offer the potential of a patient-specific therapy. Although several studies have demonstrated the ability to derive ECs with presentation of phenotypic markers, studies validating their response to hypoxia or comparing these responses relative to more mature endothelial cells are lacking. Here, we have presented data comparing the response of hiPSC-ECs and HRECs, as a mature cell control, to hypoxic environments both in vitro and in vivo.

Although the data presented here, exploring the 2D qualitative differences in hiPSC-EC and HREC expression of EC markers including CD31 and VE-Cad, found no striking difference between atmospheric and hypoxic conditions, we revealed differences in 2D proliferation for the cell types and oxygen tension conditions. Though hypoxia had no significant effect on the proliferation rate of hiPSC-ECs, the proliferation in HRECs was slowed under hypoxic conditions. These data point to the adaptability of hiPSC derivatives being similar to that of hiPSCs. Our group has previously published on the adaptability of hiPSCs to low-oxygen environments, displaying no change in proliferation and low-oxygen uptake rates under hypoxic conditions (19). hiPSC-ECs appear to have retained this phenotype of hypoxic adaptability. 
A
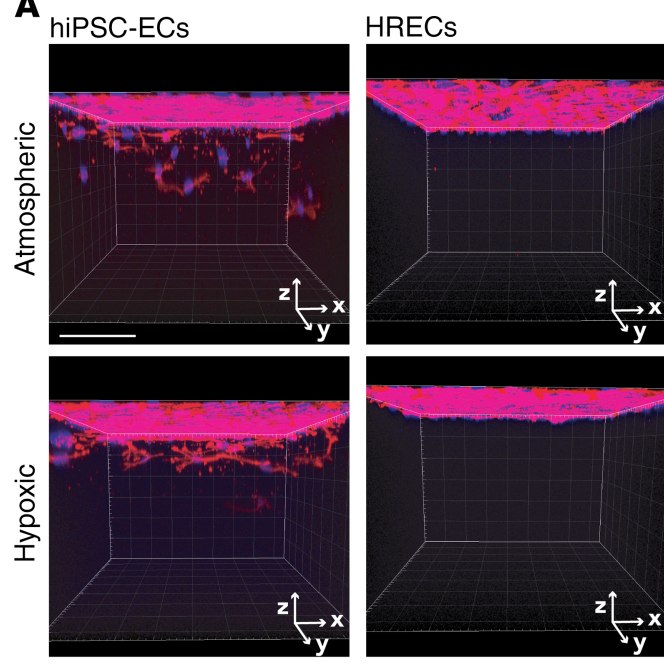

B

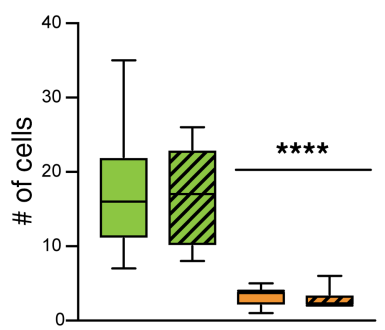

hiPSC-EC atmospheric

HREC atmospheric

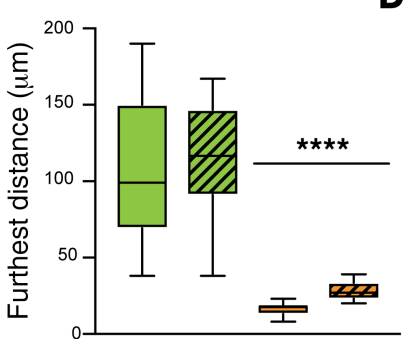

ZTh hiPS-EC hypoxic

ZI HREC hypoxic
C
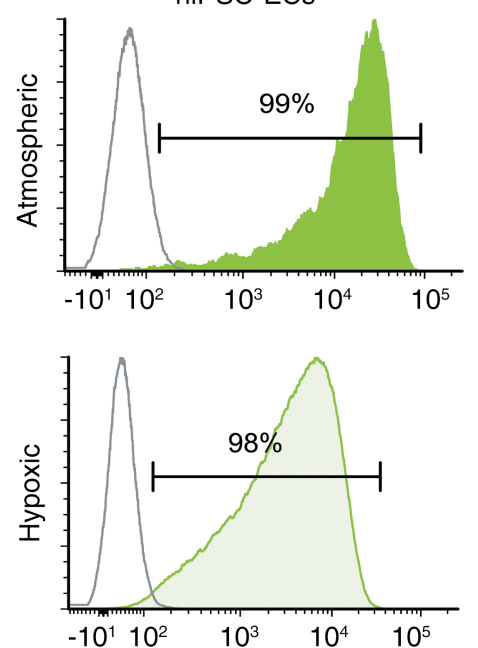

D

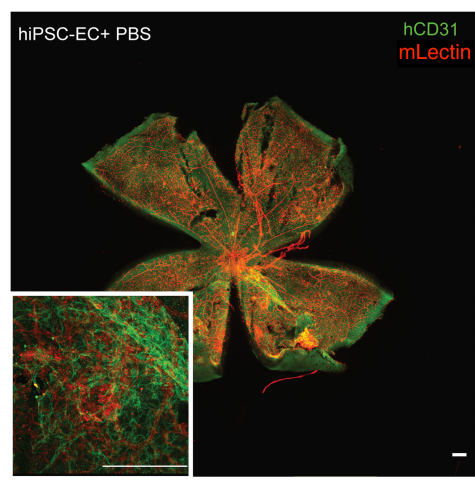

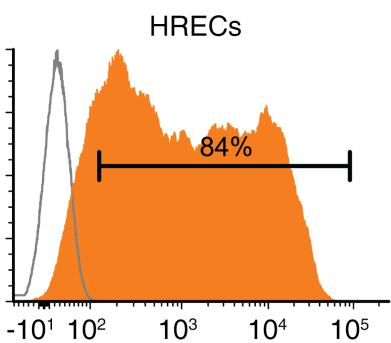
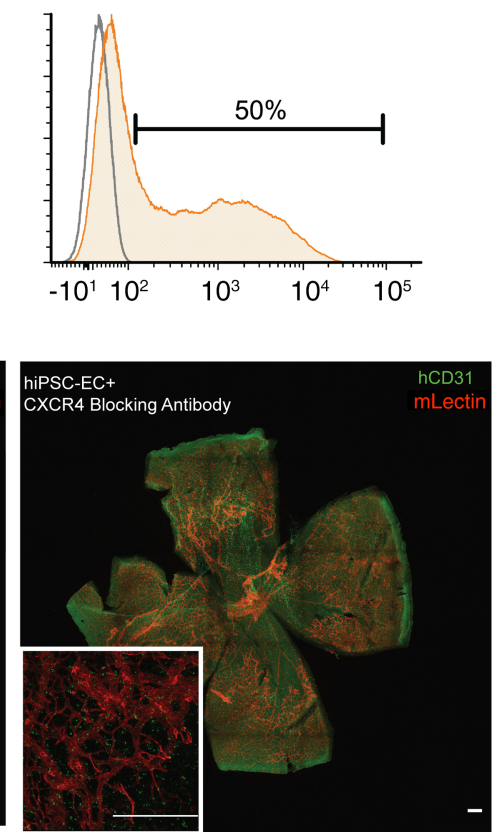

Figure 4. Activation of SDF1a/CXCR4 in hiPSC-ECs and not in HRECs. (A) Representative immunofluorescence images and (B) quantification of angiogenic invasion into collagen type I stromal cell-derived factor-1a (SDF1a) hydrogels (phalloidin in red, nuclei in blue; $n=9, n=3$ ). Tukey's multiple comparisons test was conducted to determine statistical significance. (C) Representative flow cytometry histograms for CXCR4 in human-induced pluripotent stem cell-derived endothelial cells (hiPSC-ECs) and human retinal endothelial cells (HRECs) under atmospheric and hypoxic conditions. Percentages denote percent positive cells as compared to an IgG control. (D) Representative images of mouse OIR model retinas injected with hiPSC-ECs in PBS (left; $n=4$ ) or hiPSC-ECs injected with C-X-C chemokine receptor type 4 (CXCR-4) blocking antibody (right; $n=7)$. Box represents 25 th to 75 th percentile, line at median value; whiskers represent minimum and maximum value. Significance level is set at ${ }^{* * * *} P \leq 0.0001$. Scale bars: $100 \mu \mathrm{m}$ (A); $200 \mu \mathrm{m}$ (D).

HIF1A and HIF2A are highly regulated transcription factors, undergoing constant degradation under atmospheric conditions. To understand if the differences between hiPSC-ECs and HRECs under hypoxia was HIF mediated, we quantified HIF1A and HIF2A proteins via Western blot. Although there were differences from replicate to replicate in the overall amount of HIF1A and HIF2A proteins, the fold difference within each replicate and its atmospheric and hypoxic control exhibited no significant difference. This was also further validated by the lack of difference between cell types in the mRNA levels of VEGF and $b F G F$, two well-validated HIF target genes.

We next used a collagen type I hydrogel platform to analyze the ability of hiPSC-ECs and HRECs to form vascular-like structures under hypoxia. In atmospheric and hypoxic conditions, hiPSC-ECs made complex vascular networks throughout the hydrogel, with greater length and more tubes compared with HRECs networks, quantified by a greater total volume. In comparison, HRECs appeared to have some sprouting events over the course of 48 hours in atmospheric conditions that were not apparent under hypoxic conditions. From these studies, we were able to conclude that the baseline network potential of hiPSC-ECs is greater than that of HRECs. Overall, our in vitro data have shown the adaptability and overall superiority of hiPSC-ECs to mature ECs in both 2D and 3D hypoxic environments.

In this study, the mouse OIR model was indispensable because it served as a platform to test the cellular responses to a hypoxic/ischemic tissue environment in vivo. In this model, mouse litters were placed 


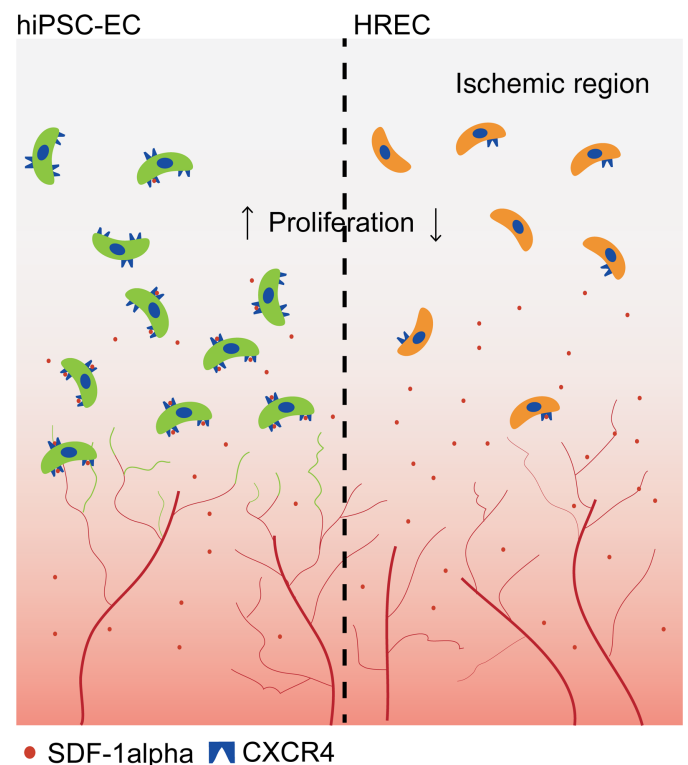

Figure 5. Proposed mechanism by which hiPSC-ECs respond to hypoxic condition in the retina. hiPSC-ECs respond to hypoxic tissue environment in a mouse model of oxygen-induced retinopathy via stromal cell-derived factor-1a (SDF1a)/ C-X-C chemokine receptor 4 (CXCR-4) axis, resulting in an integration with host vasculature compared with HRECs.

in $75 \%$ oxygen for 5 days from P7 to P12, during which time the immature blood vessels regressed (VO). The transfer of mice back to room air on P12 resulted in tissue hypoxia in the VO regions and stimulated proangiogenic responses (20), which resulted in both partial physiological revascularization of the ischemic area and a maladaptive pathologic preretinal NV that occurred anterior to the retinal tissue (21). This pattern of aberrant NV resembled that of proliferative diabetic retinopathy and other ischemic retinopathies. We injected the hiPSC-ECs or HRECs on P12, immediately after the mouse litters were removed from $75 \%$ oxygen. Hence, the injected cells were exposed to an environment where the hypoxia-induced proangiogenic responses were initiating. On P17, the hiPSC-ECs showed more widespread (volume of hiPSC-EC staining) and a greater degree of (percentage) colocalization with host lectin-stained vessels than the HRECs. This effect of superior colocalization was observed in two strains of mice, the immunodeficient NOD/ SCID mice and the immunocompetent C57BL/6 mice. This result is in accordance with a previous study in which hiPSC-ECs integrated into developing vasculatures in zebrafish, whereas human umbilical vein ECs failed to integrate into zebrafish blood vessels (22). Interestingly, although the distance for cellular homing is short with intravital injection, homing to the host blood vessels is still needed. Our data show that hiPSC-ECs home effectively to integrate with host vasculature, whereas HRECs fail to integrate into the host vasculature, further highlighting the homing challenge.

Next, we sought to understand the specific cues governing homing of the injected cells. We were especially interested in extending the phenotypic comparison of the two cell types with respect to angiogenic response to SDF1a, a chemokine that is known to play a major role in EC recruitment and be upregulated in OIR (23). First, we found that hiPSC-ECs highly expressed SDF1a receptor, CXCR4, compared with HRECs. Importantly, the hiPSC-ECs exhibited a striking contrast in the $3 \mathrm{D}$ angiogenic response to SDF1a, both under atmospheric and hypoxic conditions. This directly correlated with the much higher levels of CXCR4 expression on the cell surface. Interestingly, a previous study demonstrated the inability of hiPSC-EC to home to an ischemic site in the hind limb ischemia model. These cells exhibited a paucity of cell surface levels of CXCR4 that was associated with absent chemotactic response to SDF1a (24). In our study, the cells were locally injected into the site of injury via intravitreal injections, and thus the required distance for the cells to travel is much shorter than with systemic injection: this approach for local administration is now commonly used in the clinical setting for retinal disease processes. Hence, our in vivo system is a much more closed system, closely resembling the experimental settings of the in vitro system. Although the distance for cellular homing is shorter with local administration, specific homing to injured vasculature, and the specific cues governing this homing, remains an important challenge for the field. 
Based on our results showing higher CXCR4 expression in hiPSC-ECs alongside increased proliferation compared with HRECs, hiPSC-ECs are likely better equipped to respond to SDF1a gradient, resulting in much more pronounced colocalization with host vasculature (Figure 5). Importantly, treatment of hiPSC-ECs with CXCR4 neutralizing antibody completely abolished their colocalization with host retinal vasculature. This suggests that even with local delivery of hiPSC-ECs, the SDF1a/CXCR4 signaling axis in hiPSC-EC is still pivotal for homing and integration in the ischemic tissue site. Following recruitment and integration with the host vasculature and subsequent revascularization, analyzing the persistence of hiPSC-ECs in the recovering retina and its effect on therapeutic outcome in OIR as well as in other disease models such as diabetic retinopathy will be important next steps toward realizing therapeutics.

Overall, the results from this study provide an important first step toward the development of cellbased strategy for the treatment of ischemic retinopathies, such as for patients with DR, with a possibility of procuring reprogrammed ECs from patients' autologous source (25). Given the importance of homing, integration, and angiogenesis in a hypoxic setting, these results could also extend to revascularization in other ischemic settings, including stroke.

\section{Methods}

Human pluripotent stem cell expansion and differentiation. C1-2 hiPSC(26) were maintained on Vitronectin-coated plates (Thermo Fisher Scientific) and supplemented with Essential 8 media (Thermo Fisher Scientific). hiPSCs were differentiated as described in Smith et al. (27). Briefly, differentiation was induced on hiPSCs cultured to $60 \%$ to $80 \%$ confluency with Essential 6 medium (Thermo Fisher Scientific) supplemented with $6 \mu \mathrm{M}$ CHIR (STEMCELL Technologies), with media changed daily over 48 hours. After 48 hours, cells were digested in TrypLE Express (Thermo Fisher Scientific) and seeded on collagen type I-coated plates at $2 \times 10^{4} \mathrm{cells} / \mathrm{cm}^{2}$ in EC differentiation media that contained Endothelial Cell Growth Medium (ECGM; Promocell) supplemented with $10 \mu \mathrm{M}$ SB-431542 (Cayman Chemical Company) and $50 \mathrm{ng} / \mathrm{mL}$ VEGF (R\&D Systems), with additional supplementation if $10 \mu \mathrm{M}$ Y-27632 for the first 24 hours. After the first 24 hours, media was changed every other day for additional 6 days.

Isolation and expansion of hiPSC-derived ECs. CD31-expressing cells were isolated via magnetic-activated cell sorting (MACS; Miltenyi Biotec Bergisch Gladbach), following the manufacturer's protocol, on day 8 of differentiation. After washing once with $1 \times$ PBS (Thermo Fisher Scientific), cells were harvested with TrypLE Express and resuspended in MACS buffer (0.5 EDTA [MilliporeSigma] and 0.5\% BSA [MilliporeSigma] in PBS). Cells were then incubated with $10 \mu 1$ of PE-conjugated anti-human CD31 (BD Biosciences) for 10 minutes at $4^{\circ} \mathrm{C}$. After incubation, unbound primary antibody was removed by washing twice with MACS buffer. Next, $20 \mu \mathrm{l}$ of anti-PE microbeads (Miltenyi Biotec Bergisch Gladbach) were added to $80 \mu \mathrm{l}$ of cells suspended in MACS buffer and incubated for an additional 15 minutes at $4^{\circ} \mathrm{C}$. Cells were washed once with MACS buffer and separated using the MS MACS separation column (Miltenyi Biotec Bergisch Gladbach). After separation, CD31 and VE-Cad enrichment was confirmed using flow cytometry as previously (27). Finally, CD $31^{+}$cells were seeded on collagen type IV-coated plates and maintained in EC differentiation media.

Primary cell culture and hypoxic cell culture. HRECs (Cell Systems) were cultured in Endothelial Cell Growth Medium-2 BulletKit (Lonza) or Complete Classic Medium (Cell Systems) in a humidified incubator at $5 \% \mathrm{CO}_{2}$, with medium changed every 48 hours. Plates were coated with collagen type I before plating and stored long term at $4^{\circ} \mathrm{C}$. For hypoxic experiments, cells were cultured for 24 hours in either Modular Incubator Chambers (Billups-Rothenberg) flushed with a $1 \% \mathrm{O}_{2} / 5 \% \mathrm{CO}_{2}$ gas mixture or a humidified incubator within the C-Shuttle glove box (BioSpherix Parish) set to $1 \% \mathrm{O}_{2}$.

Immunofluorescence staining and imaging. hiPSC-ECs or HRECs were cultured on coverslips and fixed in $4 \%$ formaldehyde after hypoxic or atmospheric treatment. Formaldehyde was removed and the samples were washed with $1 \times$ PBS. Cells were then permeablized with $0.1 \%$ Triton X-100 (Sigma-Aldrich) for 10 minutes. Samples were washed and then incubated with $1 \%$ BSA solution for 1 hour at room temperature. After blocking, samples were washed with PBS and incubated with primary antibodies (see Supplemental Table 1) in antibody diluent (DAKO) overnight at $4^{\circ} \mathrm{C}$ and then incubated with secondary antibodies in antibody diluent (DAKO) for 1 hour at room temperature. Samples were then washed 3 times with $1 \times$ PBS, incubated for 3 minutes with DAPI (Roche Diagnostics), and then washed and placed onto glass slides using mounting media (Dako). Cells and hydrogels were imaged using a Zeiss Laser Scanning Microscope 780 confocal microscope. 
qRT-PCR gene expression analysis. Total RNA was extracted using TRIzol reagent (Thermo Fisher Scientific) and purified using the Direct-zol RNA Miniprep Kit (Zymo Research). RNA quality was assessed using a NanoDrop spectrophotometer (Thermo Fisher Scientific). cDNA was generated using Moloney murine leukemia virus reverse transcriptase and oligo(dT) primers (Promega) per the manufacturer's protocol. The TaqMan Universal PCR Master Mix and Gene Expression Assay (Thermo Fisher Scientific) was used for VEGFA and FGF2. TaqMan PCR was performed using the StepOne Real-Time PCR System (Thermo Fisher Scientific). The comparative computerized tomography method was used to calculate the amplification difference between samples as normalized to the endogenous control gene TBP.

Flow cytometry. Cells were harvested for analysis using TrypLE (Invitrogen) disassociation buffer and collected in $100 \mu \mathrm{L}$ of $0.1 \%$ BSA. After collection, cells were incubated with PE-conjugated CXCR 4 antibody (BioLegend) for 30 minutes on ice. Cells were washed 3 times with $0.1 \%$ BSA and passed through a $40-\mu \mathrm{m}$ cell strainer. Flow analysis was conducted on a BD FACSCanto flow cytometer. Following the manufacturer's instructions, dead cell populations were gated out with forward-side scatter plots. To determine levels of expression, all analyses were conducted using IgG-PE or IgG-FITC (BD Biosciences) isotype controls. Following the manufacturer's instructions, dead cell populations were gated out with forward-side scatter plots. All analyses were conducted using FCS Express 6 Flow (De Novo Software). For analysis of cells under hypoxia, cell culture and all staining steps were carried out in a C-Shuttle glove box with ProOX 360 gas controller (BioSpherix) with $\mathrm{CO}_{2}$ levels maintained at $5 \%$ and $\mathrm{O}_{2}$ maintained at $0.8 \%$.

Western blot. Cells were lysed using RIPA Buffer (Thermo Fisher Scientific) with $1 \times$ Protease and Phosphatase Inhibitor Cocktail (Thermo Fisher Scientific). Protein was quantified using the BCA Assay (Thermo Fisher Scientific). Protein (20-25 $\mu \mathrm{g}$ ) from each sample was boiled at $95^{\circ} \mathrm{C}$ for 5 minutes and then loaded into a $4 \%$ to $12 \%$ Bis-Tris Protein Gel (Thermo Fisher Scientific). Proteins were transferred to a PVDF membrane (Bio-Rad) via wet transfer in a Bio-Rad Criterion system for 60 minutes. Total protein was quantified using a Ponceau S stain and imaged using the ChemiDoc XRS+ System (BioRad). Membranes were blocked in 5\% milk for 1 hour and then incubated in primary antibody (see Supplemental Table 1 for specific antibody information). Membranes were washed 3 times for 10 minutes in Tris-buffered saline with $0.1 \%$ Tween-20 (TBST; Sigma-Aldrich) and then incubated for 1 hour with anti-rabbit IgG, HRP-linked antibody (Cell Signaling Technologies) with gentle agitation. The membrane was washed 3 times with TBST; to visualize protein, Clarity Western ECL Substrate (Bio-Rad) was added, and membranes were imaged using the ChemiDoc XRS+ System (Bio-Rad). Blots were analyzed using Image Lab software (Bio-Rad) and bands were normalized to $\beta$-actin expression.

$3 D$ collagen gel assay and immunofluorescence staining. Collagen gels were formed preciously as described (28). To prepare $1 \mathrm{~mL}$ of collagen gel solution, 800,000 cells were resuspended in $400 \mu \mathrm{L}$ of Medium 199 $(1 \times), 40 \mu \mathrm{L}$ of Medium $199(10 \times)$, and $350 \mu \mathrm{L}$ of $7.1 \mathrm{mg} / \mathrm{mL}$ Rat Tail Collagen I (Corning). The $\mathrm{pH}$ of the solution was adjusted by titrating $1 \mathrm{M} \mathrm{NaOH}$ up to $10 \mu \mathrm{L}$. Of the mixture, $56 \mu \mathrm{L}$ was added to the wells of a 96-well plate. Gels were polymerized at $37^{\circ} \mathrm{C}$, and ECGM, supplemented with $50 \mathrm{ng} / \mathrm{mL}$ of VEGF, was added after 30 minutes. For hypoxia studies, gels were cultured in a hermitically sealed chamber flushed with $1 \% \mathrm{O}_{2} / 5 \% \mathrm{CO}_{2} / \mathrm{N}_{2}$ balance for 48 hours. After 48 hours, gels were fixed in $2 \%$ formaldehyde for 20 minutes. Formaldehyde was removed and cells were washed 3 times in $1 \times$ PBS. Gels were incubated in 1\% Triton-X 100 (Sigma-Aldrich) for 10 minutes and then rinsed in $1 \times$ PBS in 30-minute intervals. After polymerization, gels were blocked in 10\% BSA solution for 1 hour at room temperature. After 1 hour, cells were incubated with a conjugated phalloidin probe for 2 hours at room temperature. Cells were rinsed with a $0.05 \%$ TWEEN 20 (Sigma-Aldrich) solution and stored in unsupplemented $1 \times$ PBS until imaged. Networks were quantified using the Imaris software Filament package.

SDF1a migration assay using collagen gels. Collagen gels were prepared as described above, with the addition of $100 \mathrm{ng} / \mathrm{mL}$ SDF1a (R\&D Systems Inc.). We seeded 75,000 cells hiPSC-ECs or HRECs on the top of polymerized collagen gels in ECGM media and invasion was allowed to occur over 24 hours in either atmospheric or hypoxic conditions. After 24 hours, gels were fixed, stained for Phalloidin and DAPI, and imaged as described above. The migration of cells into gels were analyzed for the number of cells invading and the distance from the top using the Imaris software Spots package. Mouse model of OIR NOD. CB17-Prkdcscid/J (NOD/SCID, Jackson laboratory) or C57BL/6J (Jackson laboratory) mice were used for all experiments. Mice were subjected to 75\% oxygen from P7 to P12 (Biospherix A-15274-P, ProOx P110 coupled to an oxygen sensor E702, Biospherix). Mice were injected with either HRECs (50,000 cells/eye, Cell Systems) or hiPSCs (50,000 cells/eye) in 1 eye via intravitreal injection (PLI-100A Pico-liter injector, 
Warner Instruments) on P12. The contralateral eyes were injected with PBS as controls. Retinal tissues were harvested at specified time points. In a subset of C57BL/6J mice, hiPSC-ECs (50,000 cells/eye) were injected in the presence or absence of CXCR4 blocking antibody (R\&D Systems). The hiPSCs were preincubated in CXCR4 blocking antibody $(0.25 \mathrm{mg} / \mathrm{mL})$ for 30 minutes and resuspended in CXCR4 antibody solution at $0.5 \mathrm{mg} / \mathrm{mL}$ before intravitreal injection. hiPSC-ECs resuspended in PBS were used as controls.

Retina flat mount and immunostaining. For flat-mount staining, eyes were enucleated and immediately fixed in 4\% PFA for 30 minutes, then a hole was created in the cornea using a 30-gauge needle and fixed for additional 90 minutes in 4\% PFA. After cornea and lens were carefully removed, retina was carefully removed from the eye cup using dissection forceps and dissecting micro scissors. The isolated retinas were blocked for 2 hours using 10\% normal goat serum containing $0.3 \%$ Triton $\mathrm{X}-100$. The retinas were then incubated with Alexa Fluor 594-conjugated Griffonia simplicifolia isolectin B4 (Invitrogen) overnight at $4^{\circ} \mathrm{C}$ in PBS containing $0.3 \%$ Triton X-100 and $100 \mu \mathrm{M} \mathrm{CaCl}_{2}$. Some retinas were incubated with primary antibody (see Supplemental Table 1) overnight at $4^{\circ} \mathrm{C}$ to stain the injected HRECs or hiPSCs. Retinas were imaged using confocal microscopy, and avascular area and neovascular tufts were quantified by comparing the number of pixels in the area of $\mathrm{VO}(\mathrm{VO} \%)$ or neovascular tufts $(\mathrm{NV} \%)$ with the total number of pixels in the retina, as described previously (29). Quantitation was performed in a masked fashion. Mice with body weight lower than $5 \mathrm{~g}$ (P17) were excluded from analysis.

Statistics. For all experiments, " $n$ " denotes technical replicates, whereas " $N$ " represents biological replicates. Unless otherwise indicated, analyses were performed in triplicate samples; $N$ is indicated for each experiment throughout the figure legends. Two-tailed $t$ test or Tukey's multiple comparisons test was performed to determine significance. All graphs were drawn using GraphPad Prism 6. A $P$ value of less than or equal to 0.05 was considered significant.

Study approval. Animal studies were approved by the Institutional Animal Care and Use Committee of the Johns Hopkins University School of Medicine. All procedures involving animals were conducted in accordance with the Association for Research in Vision and Ophthalmology Statement for the Use of Animals in Ophthalmic and Vision Research.

\section{Author contributions}

BM, SG, and ED conceived the study, designed experiments, interpreted the results, and wrote the manuscript. HC designed experiments, interpreted the results, and wrote the manuscript. YL, LZ, MJL, and GL performed experiments and analyzed data.

\section{Acknowledgments}

We thank XinYi Chan and Michael Blatchley for helpful discussions throughout the course of this work. This work was supported by the NRSA F31 predoctoral fellowship F31HL143972 (to BM), from the NIH/ National Heart, Lung, and Blood Institute, and Maryland Stem Cells Research Fund Grant MSCRFI-2784 (to SG) as well as research grants from the National Institutes of Health (EY022383 and EY022683; to EJD) and Core Grant P30EY001765, Imaging and Microscopy Core Module.

Address correspondence to: Elia J. Duh, Wilmer Ophthalmological Institute, Johns Hopkins University School of Medicine, 400 North Broadway, Smith 3011, Baltimore, Maryland 21231, USA. Phone: 410.614.3388; Email: eduh@jhmi.edu. Or to: Sharon Gerecht, Department of Chemical and Biomolecular Engineering, Institute for NanoBioTechnology, Johns Hopkins University, 3400 North Charles Street Croft Hall 110, Baltimore, Maryland 21231, USA. Phone: 410.516.2846; Email: gerecht@jhu.edu.

1. Calvani M, Rapisarda A, Uranchimeg B, Shoemaker RH, Melillo G. Hypoxic induction of an HIF-1 $\alpha$-dependent bFGF autocrine loop drives angiogenesis in human endothelial cells. Blood. 2006;107(7):2705-2712.

2. Manalo DJ, et al. Transcriptional regulation of vascular endothelial cell responses to hypoxia by HIF-1. Blood. 2005;105(2):659-669.

3. Sapieha P. Eyeing central neurons in vascular growth and reparative angiogenesis. Blood. 2012;120(11):2182-2194

4. Ii M, Kawamoto A, Masuda H, Asahara T. Vascular regeneration therapy: endothelial progenitor cells for ischemic diseases. In: Steinhoff G, ed. Regenerative Medicine - from Protocol to Patient: 5 Regenerative Therapies II. Cham: Springer International Publishing; 2016:35-57

5. Yiu KH, Tse HF. Specific role of impaired glucose metabolism and diabetes mellitus in endothelial progenitor cell characteristics and function. Arterioscler Thromb Vasc Biol. 2014;34(6):1136-1143.

6. Loomans CJ, et al. Endothelial progenitor cell dysfunction: a novel concept in the pathogenesis of vascular complications of 
type 1 diabetes. Diabetes. 2004;53(1):195-199.

7. Wang L, et al. Human induced pluripotent stem cells derived endothelial cells mimicking vascular inflammatory response under flow. Biomicrofluidics. 2016;10(1):014106.

8. Stahl A, et al. The mouse retina as an angiogenesis model. Invest Ophthalmol Vis Sci. 2010;51(6):2813-2826.

9. Patel SN, Gangaputra S, Sternberg P, Kim SJ. Prophylaxis Measures for Post-Injection Endophthalmitis. Surv Ophthalmol. 2020;null(1):S0039-6257(20)30001-1.

10. Xie Y, Fan Y, Xu Q. Vascular Regeneration by Stem/Progenitor Cells. Arterioscler Thromb Vasc Biol. 2016;36(5):e33-e40.

11. NIDDK. Diabetes Statistics. NIH website. https://www.niddk.nih.gov/health-information/health-statistics/diabetes-statistics. Accessed July 5, 2019.

12. Husi $\mathrm{H}$, et al. Proteome-based systems biology analysis of the diabetic mouse aorta reveals major changes in fatty acid biosynthesis as potential hallmark in diabetes mellitus-associated vascular disease. Circ Cardiovasc Genet. 2014;7(2):161-170.

13. Abcouwer SF, Gardner TW. Diabetic retinopathy: loss of neuroretinal adaptation to the diabetic metabolic environment. Ann $N Y$ Acad Sci. 2014;1311:174-190.

14. MacAskill MG, et al. Robust revascularization in models of limb ischemia using a clinically translatable human stem cell-derived endothelial cell product. Mol Ther. 2018;26(7):1669-1684

15. Cho SW, et al. Improvement of postnatal neovascularization by human embryonic stem cell derived endothelial-like cell transplantation in a mouse model of hindlimb ischemia. Circulation. 2007;116(21):2409-2419.

16. Clayton ZE, et al. Induced pluripotent stem cell-derived endothelial cells promote angiogenesis and accelerate wound closure in a murine excisional wound healing model. Biosci Rep. 2018;38(4):BSR20180563.

17. Kim KL, Song SH, Choi KS, Suh W. Cooperation of endothelial and smooth muscle cells derived from human induced pluripotent stem cells enhances neovascularization in dermal wounds. Tissue Eng Part A. 2013;19(21-22):2478-2485.

18. Shen YI, et al. Engineered human vascularized constructs accelerate diabetic wound healing. Biomaterials. 2016;102:107-119.

19. Abaci HE, Truitt R, Luong E, Drazer G, Gerecht S. Adaptation to oxygen deprivation in cultures of human pluripotent stem cells, endothelial progenitor cells, and umbilical vein endothelial cells. Am J Physiol, Cell Physiol. 2010;298(6):C1527-C1537.

20. Kim CB, D'Amore PA, Connor KM. Revisiting the mouse model of oxygen-induced retinopathy. Eye Brain. 2016;8:67-79.

21. Liu CH, Wang Z, Sun Y, Chen J. Animal models of ocular angiogenesis: from development to pathologies. FASEB J. 2017;31(11):4665-4681.

22. Orlova VV, et al. Functionality of endothelial cells and pericytes from human pluripotent stem cells demonstrated in cultured vascular plexus and zebrafish xenografts. Arterioscler Thromb Vasc Biol. 2014;34(1):177-186.

23. Lima e Silva R, et al. The SDF-1/CXCR4 ligand/receptor pair is an important contributor to several types of ocular neovascularization. FASEB J. 2007;21(12):3219-3230.

24. Huang NF, et al. Chemotaxis of human induced pluripotent stem cell-derived endothelial cells. Am J Transl Res. 2013;5(5):510-520

25. Chan XY, et al. Three-dimensional vascular network assembly from diabetic patient-derived induced pluripotent stem cells. Arterioscler Thromb Vasc Biol. 2015;35(12):2677-2685.

26. Wen Z, et al. Synaptic dysregulation in a human iPS cell model of mental disorders. Nature. 2014;515(7527):414-418

27. Sokic S, Papavasiliou G. Controlled proteolytic cleavage site presentation in biomimetic PEGDA hydrogels enhances neovascularization in vitro. Tissue Eng Part A. 2012;18(23-24):2477-2486.

28. Kusuma S, Macklin B, Gerecht S. Derivation and network formation of vascular cells from human pluripotent stem cells. Methods Mol Biol. 2014;1202:1-9.

29. Connor KM, et al. Quantification of oxygen-induced retinopathy in the mouse: a model of vessel loss, vessel regrowth and pathological angiogenesis. Nat Protoc. 2009;4(11):1565-1573. 